\title{
Iterative Reconstruction for Quantitative Tissue Decomposition in Dual-Energy CT
}

\author{
Maria Magnusson ${ }^{1,2,3}$, Alexandr Malusek ${ }^{2,3,4}$, \\ Arif Muhammad ${ }^{2}$, and Gudrun Alm Carlsson ${ }^{2,3}$ \\ 1 Dept. of Electrical Engineering, \\ 2 Dept. of Medical and Health Sciences, Radiation Physics, \\ ${ }^{3}$ Center for Medical Image Science and Visualization (CMIV), \\ Linköping University, SE-581 83 Linköping, Sweden \\ 4 Dept. of Radiation Dosimetry, Nuclear Physics Institute AS CR, v.v.i., \\ Na Truhlarce 39/64, 18086 Praha 8, Czech Republic \\ \{maria.magnusson, alexandr.malusek, gudrun.alm.carlsson\}@liu.se, \\ muhammad_arif57@hotmail.com
}

\begin{abstract}
Quantitative tissue classification using dual-energy CT has the potential to improve accuracy in radiation therapy dose planning as it provides more information about material composition of scanned objects than the currently used methods based on single-energy CT. One problem that hinders successful application of both single- and dualenergy $\mathrm{CT}$ is the presence of beam hardening and scatter artifacts in reconstructed data. Current pre- and post-correction methods used for image reconstruction often bias CT numbers and thus limit their applicability for quantitative tissue classification.

Here we demonstrate simulation studies with a novel iterative algorithm that decomposes every soft tissue voxel into three base materials: water, protein and adipose. The results demonstrate that beam hardening artifacts can effectively be removed and accurate estimation of mass fractions of all base materials can be achieved.

In the future, the algorithm may be developed further to include segmentation of soft and bone tissue and subsequent bone decomposition, extension from 2-D to 3-D and scatter correction.
\end{abstract}

Keywords: Iterative reconstruction, Dual energy CT, Tissue classification, Tissue composition, Tissue decomposition.

\section{Introduction}

\subsection{Information Attainable from CT Imaging}

Computed tomography (CT) measures spatial distribution of the linear attenuation coefficient, $\mu(x, y, z)$, [1]. The reconstructed $\mu$-values are affected by quantum noise, scatter, and beam hardening. Scatter and beam hardening lead to cupping and streak artifacts in reconstructed images. Pre-processing with a water beam hardening correction algorithm [1] is used in practice. For head imaging,

A. Heyden and F. Kahl (Eds.): SCIA 2011, LNCS 6688, pp. 479 488, 2011.

(C) Springer-Verlag Berlin Heidelberg 2011 
post-processing to remove severe streaks and cupping caused by beam hardening in bone is performed. In this case, however, the received $\mu$-values for bone are inaccurate. It is generally accepted that a more complete suppression of scatter and beam hardening artifacts can only be achieved by iterative image reconstruction algorithms.

\subsection{Recent Technical Developments in CT Imaging}

CT techniques have developed rapidly in recent years. To speed up image acquisition, spiral and multi-slice imaging techniques are used. Helical scanning with multi-row detectors increases the speed even further and provides reconstructed volumes, see for example [2].

Dual Energy CT (DECT) with two rotating X-ray tubes was introduced by Siemens for heart scanning. Later, by using two different tube voltages, it was used to improve segmentation of anatomical structures with tissue compositions too close to be discriminated with conventional CT scanners [3].

\subsection{Tissue Classification Using Single-Energy CT Scans}

In single-energy CT, the historically first tissue classification method was performed by assigning the linear attenuation coefficient values into groups (for instance bone, soft tissue, etc.) delimited by threshold values. A more elaborate method currently used in clinical practice was developed by [4]. They assumed that each tissue was a mixture of two base materials and derived formulas for the determination of weight fractions of these two materials. The authors suggested that all soft tissues could be expressed as a weighted mixture of three materials (water, protein and adipose) but their single-energy technique did not allow tissues to be expressed as a combination of three materials.

\subsection{Tissue Classification Using Dual-Energy CT Scans}

In [5], it was demonstrated that DECT can be used to determine electron densities and effective atomic numbers. In [6] it was showed that DECT can be used to quantify mass fractions of three materials (water, hydroxyapatite and aqueous iron nitrate). In [7] the method was applied for the determination of (i) iron content in liver composed of soft tissue, fat, and iron, and (ii) bone-mineral density in a trabecular bone composed of calcium hydroxyappitite (CaHA), yellow- and red-marrow. All these applications may help in non-invasive medical diagnostic methods. None of the DECT applications suggested so far, however, has considered to use the data about quantitative tissue classification for the suppression of beam hardening and scatter artifacts. In this respect, our approach is novel.

\section{Methods}

\subsection{Filtered Backprojection Reconstruction in CT}

The most common reconstruction method in CT is filtered backprojection. There exist different variants for parallel and fanbeam projection geometries, see for 


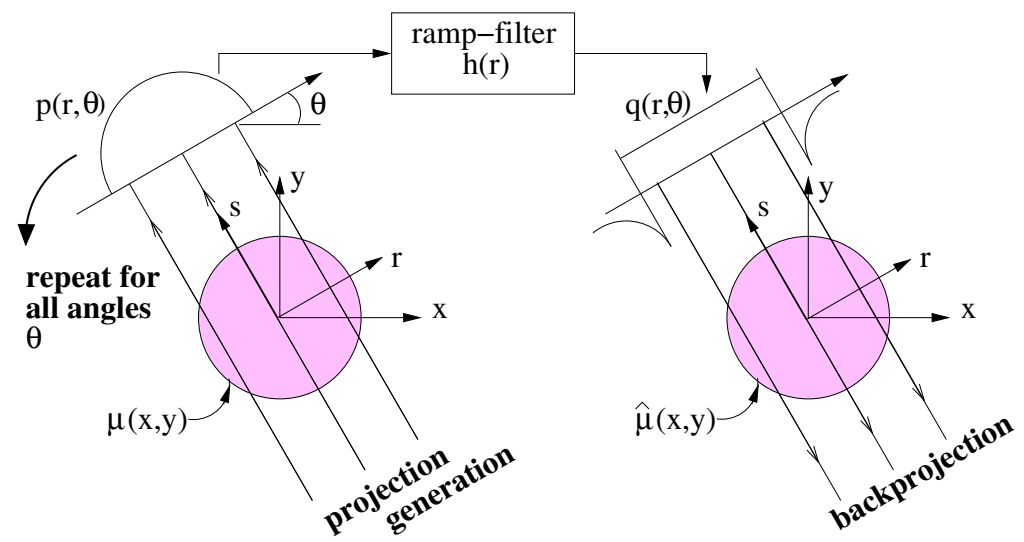

Fig. 1. Parallel computed tomography illustrated

example 1], as well as for helical geometry, see for example [2]. It is also possible to obtain parallel projections by rebinning fanbeam projections (a resorting and interpolation process). Here we describe parallel filtered backprojection, see Fig. 1. The projection generation is performed by the CT scanner and subsequent rebinning. The actual filtered backprojection reconstruction consists of ramp-filtering and backprojection. The measured attenuation coefficient data is denoted $\mu(x, y)$ and the reconstructed object is denoted $\hat{\mu}(x, y)$.

Projection generation is described by

$$
p(r, \theta)=\int_{-\infty}^{\infty} \mu(x, y) d s, \quad \text { where } \quad\left(\begin{array}{l}
x \\
y
\end{array}\right)=\left(\begin{array}{rr}
\cos \theta & -\sin \theta \\
\sin \theta & \cos \theta
\end{array}\right)\left(\begin{array}{l}
r \\
s
\end{array}\right) .
$$

The ramp-filter is applied according to

$$
q(r, \theta)=\mathcal{F}_{r}^{-1}\left[\mathcal{F}_{r}[p(r, \theta)] \cdot \mathcal{F}_{r}[h(r)]\right],
$$

where $\mathcal{F}_{r}$ denotes the Fourier transform in the $r$-direction, $\mathcal{F}_{r}^{-1}$ denotes the inverse Fourier transform, and

$$
\mathcal{F}[h(r)]=H(\rho)=\left\{\begin{array}{cr}
|\rho|, \text { if }|\rho| \leq \rho_{\max }, \\
0, \quad \text { elsewhere }
\end{array}\right.
$$

Then back-projection is applied, which means smearing of filtered projection data over the image plane according to

$$
\hat{\mu}(x, y)=\int_{0}^{\pi} q(x \cos \theta+y \sin \theta, \theta) d \theta .
$$

Note that projection and backprojection must be repeated for all angles $\theta$ in the interval $0 \leq \theta<\pi$. 


\subsection{Segmentation of Body Tissues}

A CT scan of a body part can only contain certain organs and tissues. The linear attenuation coefficient $\mu\left[\mathrm{cm}^{-1}\right]$ for different tissues can be plotted for two X-ray tube voltages, 80 and $140 \mathrm{kV}$, in a linear attenuation coefficient (LAC) diagram, see Fig. 22 In a first step, these tissues are classified using a threshold classification to for instance lung, soft, and bone tissues, as indicated in the figure. This might, however, not be sufficient. There is, for example a risk that bone marrow will be classified as soft tissue. Topologic information may then be taken under consideration, e.g. a procedure of image segmentation. In the current work, however, only soft tissues were considered. In the second step, the soft tissues are classified using the three-material decomposition method as described in the next section.

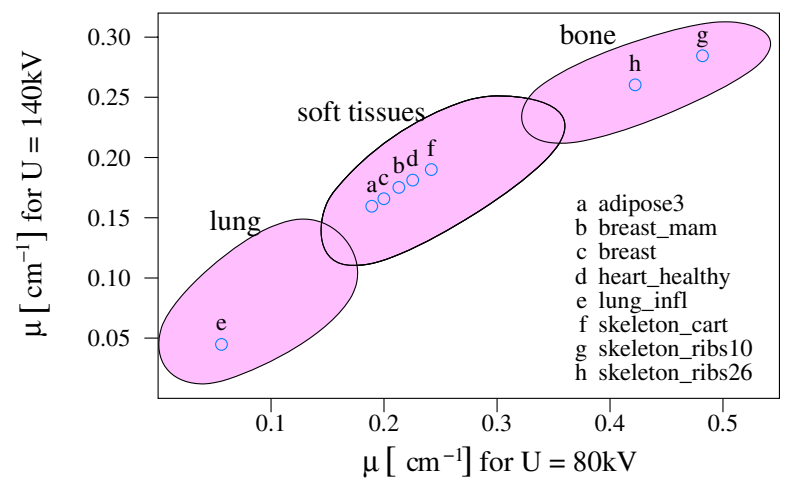

Fig. 2. Threshold classification in the LAC diagram separates lung, soft, and bone tissues. Attenuation coefficients for selected tissues defined in ICRU44 [8].

\subsection{The Three Material Decomposition method}

We extended the method by Schneider 4 to dual-energy CT (DECT) as the three-material decomposition method. It is important to choose a proper set of base materials. For our case with soft tissue decomposition we chose water, protein and adipose. Assume that the tissue consists of a mixture of these base materials with mass fractions $w_{1}, w_{2}$ and $w_{3}$, where

$$
w_{1}+w_{2}+w_{3}=1 \text {. }
$$

If the volume of the mixture is the sum of the volumes of individual components, then the density $\rho$ of the three-material mixture in a volume $V\left[\mathrm{~m}^{3}\right]$ with mass $m[\mathrm{~kg}]$ can be written

$$
\rho=\frac{m}{V}=\frac{m}{\frac{m_{1}}{\rho_{1}}+\frac{m_{2}}{\rho_{2}}+\frac{m_{3}}{\rho_{3}}}=\frac{1}{\frac{w_{1}}{\rho_{1}}+\frac{w_{2}}{\rho_{2}}+\frac{1-w_{1}-w_{2}}{\rho_{3}}} .
$$


The linear attenuation coefficient for the mixture of materials depends on the volume fractions of the three materials as

$$
\mu=w_{1} \frac{\rho}{\rho_{1}} \mu_{1}+w_{2} \frac{\rho}{\rho_{2}} \mu_{2}+w_{3} \frac{\rho}{\rho_{3}} \mu_{3}=\frac{V_{1}}{V} \mu_{1}+\frac{V_{2}}{V} \mu_{2}+\frac{V_{3}}{V} \mu_{3},
$$

where we have utilized the mixture rule and the fact that

$$
\frac{V_{1}}{V}=\frac{m_{1} \rho}{\rho_{1} m}=\frac{w_{1} \rho}{\rho_{1}},
$$

and similarly for $V_{2}$ and $V_{3}$. Using (7) and (5), $\mu\left(E_{1}\right)$ and $\mu\left(E_{2}\right)$ for energies $E_{1}$ and $E_{2}$ can be written

$$
\left\{\begin{array}{l}
\mu\left(E_{1}\right)=\rho\left(w_{1} \frac{\mu_{1}\left(E_{1}\right)}{\rho_{1}}+w_{2} \frac{\mu_{2}\left(E_{1}\right)}{\rho_{2}}+\left(1-w_{1}-w_{2}\right) \frac{\mu_{3}\left(E_{1}\right)}{\rho_{3}}\right), \\
\mu\left(E_{2}\right)=\rho\left(w_{1} \frac{\mu_{1}\left(E_{2}\right)}{\rho_{1}}+w_{2} \frac{\mu_{2}\left(E_{2}\right)}{\rho_{2}}+\left(1-w_{1}-w_{2}\right) \frac{\mu_{3}\left(E_{2}\right)}{\rho_{3}}\right) .
\end{array}\right.
$$

The mass attenuation coefficient is defined as $\mu / \rho$. Using the vector notation $\bar{M}=\left(\mu\left(E_{1}\right) / \rho, \mu\left(E_{2}\right) / \rho\right)^{T}$ for a mixture of interest (and similarly for base materials $\bar{M}_{1}, \bar{M}_{2}$, and $\bar{M}_{3}$ ), equation (9) can be written

$$
\bar{M}=w_{1}\left(\bar{M}_{1}-\bar{M}_{3}\right)+w_{2}\left(\bar{M}_{2}-\bar{M}_{3}\right)+\bar{M}_{3} .
$$

This equation is illustated in Fig. 3 .

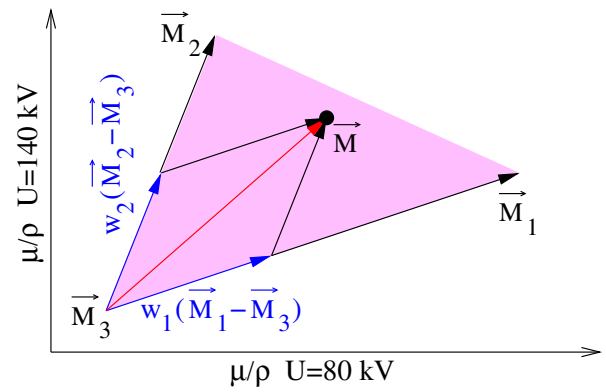

Fig. 3. Vector diagram for the three-material decomposition method

Now by combining equations (6) and (9), we get a system of linear equations,

$$
\left(\begin{array}{c}
\frac{\mu\left(E_{1}\right)-\mu_{3}\left(E_{1}\right)}{\rho_{3}} \\
\frac{\mu\left(E_{2}\right)-\mu_{3}\left(E_{2}\right)}{\rho_{3}}
\end{array}\right)+\mathbf{M}\left(\begin{array}{c}
w_{1} \\
w_{2}
\end{array}\right)=\left(\begin{array}{l}
0 \\
0
\end{array}\right)
$$

where

$$
\mathbf{M}=\left[\begin{array}{l}
\frac{\mu\left(E_{1}\right)-\mu_{1}\left(E_{1}\right)}{\rho_{1}}-\frac{\mu\left(E_{1}\right)-\mu_{3}\left(E_{1}\right)}{\rho_{3}} \frac{\mu\left(E_{1}\right)-\mu_{2}\left(E_{1}\right)}{\rho_{2}}-\frac{\mu\left(E_{1}\right)-\mu_{3}\left(E_{1}\right)}{\rho_{3}} \\
\frac{\mu\left(E_{2}\right)-\mu_{1}\left(E_{2}\right)}{\rho_{1}}-\frac{\mu\left(E_{2}\right)-\mu_{3}\left(E_{2}\right)}{\rho_{3}} \frac{\mu\left(E_{2}\right)-\mu_{2}\left(E_{2}\right)}{\rho_{2}}-\frac{\mu\left(E_{2}\right)-\mu_{3}\left(E_{2}\right)}{\rho_{3}}
\end{array}\right] .
$$

The solutions to (11) are the weight fractions $w_{1}$ and $w_{2}$ of the first two materials. The third weight fraction $w_{3}$ is obtained from (5). 


\subsection{The Iterative Reconstruction Algorithm}

The iterative reconstruction algorithm is illustrated in Fig. 4. As indicated in the figure, the iterative loop consists of two parts, one for each energy. Fuchs used a somewhat similar iterative reconstruction algorithm, which was only in one part, however. It is described and referenced in 9 . Note that the projections are reconstructed with a full ramp-filtered backprojection.

- Two sets of projections denoted $\mathbf{P}_{\mathbf{M}, \mathbf{U} \mathbf{1}}$ and $\mathbf{P}_{\mathbf{M}, \mathbf{U} \mathbf{2}}$ are measured by the CT scanner for two different X-ray spectra corresponding to X-ray tube voltages of $U_{1}$ and $U_{2}$. All other data are initialized to 0 .

- The measured projections are submitted to the filtered backprojection algorithm which computes the reconstructed images $\mu_{1}$ and $\mu_{2}$ with attenuation coefficients corresponding approximately to the effective energies $E_{1}$ and $E_{2}$ of the X-ray spectra $U_{1}$ and $U_{2}$.

- Our tissue classification method described in sections 2.2 and 2.3 (the tissue in a voxel is a mixture of three basic tissues) gives the classified reconstructed image $\mu_{\mathbf{C}}$.

- Then monoenergetic projections $\mathbf{P}_{\mathbf{E} 1}$ and $\mathbf{P}_{\mathbf{E} 2}$ at energies $E_{1}$ and $E_{2}$, and polyenergetic projections $\mathbf{P}_{\mathbf{U} \mathbf{1}}$ and $\mathbf{P}_{\mathbf{U} \mathbf{2}}$ for spectra $U_{1}$ and $U_{2}$ are calculated.

- The polyenergetic projections are then subtracted from the measured projections, giving a (small) error term. The error term is added to the monoenergetic projections and the result is submitted to the next iteration.

The error term will diminish with each iteration. The final result is the reconstructed images $\mu_{1}$ and $\mu_{2}$ for energies $E_{1}$ and $E_{2}$ and the $\mu_{\mathbf{C}}$ image containing classified tissue voxels. The $\mu_{1}, \mu_{2}$ and $\mu_{\mathbf{C}}$ images will be free from beam hardening distortions.

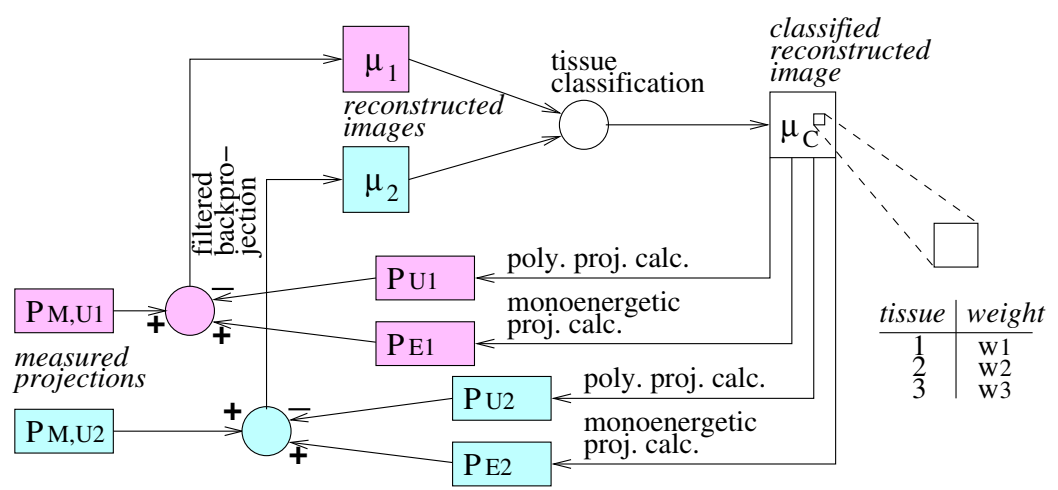

Fig. 4. Our iterative reconstruction algorithm. After a certain number of iterations, the $\mu_{1}$ and $\mu_{2}$ images will contain reconstructions corresponding to energies $E_{1}$ and $E_{2}$, respectively, and the $\mu_{C}$ image will contain classified tissue voxels. The three images will be free from beam hardening distortions. 


\section{Experiments}

\subsection{Considerations for the Iterative Reconstruction Algorithm}

For simulation of measured X-ray projections the MATLAB/C program take was used [10]. In the simulation, a fan-beam CT geometry with flat detector, 280 projections per rotations, 256 detector elements, and a fan-beam angle of $13^{\circ}$ was used. The source-to-isocenter distance was $1 \mathrm{~m}$. The fan-beam projections were rebinned to parallel projections before they were used in the iterative loop. The energy spectra were produced by x-ray tube voltages of $80 \mathrm{kV}$ and $140 \mathrm{kV}$ (the latter voltage was used in combination with an additional Sn filter) provided by Siemens under a non-disclosure agreement. Their principal appearances are shown in Fig. 5 , The calculation of projections on the classified reconstructed image was performed using the method in [11].

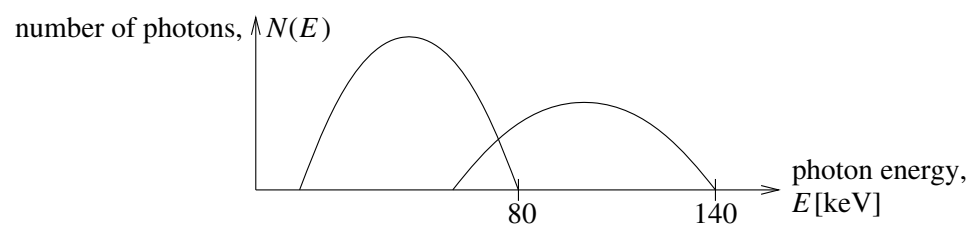

Fig. 5. The principal appearances of two spectra, a $80 \mathrm{kV}$ and a $140 \mathrm{kV}$ with Sn filter

\subsection{The Mathematical Phantom}

A mathematical phantom was used in the experiment. It consisted of a large circular water disc $R_{0}$ of diameter $40 \mathrm{~cm}$, with five small circular regions $R_{1}$ to $R_{5}$ of size $5 \mathrm{~cm}$, numbered from top-to-bottom and from left-to-right, see Fig. 6. The regions were composed of three base materials: water, protein and adipose and their mixtures with known mass fractions as given in Table 1 .

Table 1. Base materials mass fractions in different regions of the phantom

\begin{tabular}{|c|c|c|c|c|c|}
\hline$R_{0}$ & $R_{1}$ & $R_{2}$ & $R_{3}$ & $R_{4}$ & $R_{5}$ \\
\hline $100 \%$ Water & $100 \%$ Protein & $\begin{array}{ll}25 \% & \text { Protein } \\
75 \% & \text { Adipose }\end{array}$ & $\begin{array}{ll}40 \% & \text { Protein } \\
30 \% & \text { Adipose } \\
30 \% & \text { Water }\end{array}$ & 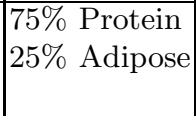 & $100 \%$ Adipose \\
\hline
\end{tabular}

\subsection{Linear Attenuation Coefficients and Effective Energies}

To generate projections through the mathematical phantom, the X-ray attenuation values of each material were calculated by using linear attenuation coefficients $\mu(E)=\rho\left[\sigma_{C o}(E)+\sigma_{I n}(E)+\sigma_{P h}(E)\right]$, where $\rho\left[\mathrm{g} / \mathrm{cm}^{3}\right]$ is the density of the 
material and $\sigma\left[\mathrm{cm}^{2} / \mathrm{g}\right]$ is the mass attenuation coefficient of the coherent scattering (Rayleigh scattering), incoherent scattering (Compton scattering) and the photoelectric effect, respectively, see e.g. [10]. The mass attenuation coefficients for water, protein and adipose were obtained from [12. The effective attenuation coefficient $\mu_{m E}\left[\mathrm{~m}^{-1}\right]$ for water was calculated as energy-fluence weighted linear attenuation coefficient for water,

$$
\mu_{m E}=\left(\int_{0}^{E_{\max }} E N(E) \mu(E) d E\right) /\left(\int_{0}^{E_{\max }} E N(E) d E\right) .
$$

Then, using the $\mu(E)$-curve for water, the energy value corresponding to $\mu_{m E}$ for water, was taken as the effective energy $E_{\text {eff }}$. Then the effective attenuation coefficients for adipose and protein were taken as $\mu\left(E_{\text {eff }}\right)$ in the attenuation curves for adipose and protein, respectively, see Table 2 .

Table 2. Effective energies and linear attenuation coefficients for the two spectra

\begin{tabular}{|c|c|c|c|c|}
\hline $\begin{array}{c}\text { X-ray } \\
\text { spectrum }\end{array}$ & $\begin{array}{c}\text { Effective } \\
\text { energies, } E_{\text {eff }}[\mathrm{keV}]\end{array}$ & $\begin{array}{c}\mu_{m E}[1 / \mathrm{m}] \\
\text { for Water }\end{array}$ & $\begin{array}{c}\mu_{m E}[1 / \mathrm{m}] \\
\text { for Protein }\end{array}$ & $\begin{array}{c}\mu_{m E}[1 / \mathrm{m}] \\
\text { for Adipose }\end{array}$ \\
\hline $80 \mathrm{kV}$ & 49.9 & 22.69 & 28.15 & 20.79 \\
$140 \mathrm{kV}+\mathrm{Sn}$ & 88.5 & 17.73 & 22.70 & 16.93 \\
\hline
\end{tabular}

Table 3. Quantitative evaluation of weight fractions measured in \%

\begin{tabular}{|c|rrr|rrr|rrr|}
\hline & \multicolumn{2}{|c|}{ True values } & \multicolumn{2}{|c|}{ After } & 0 iter. & \multicolumn{2}{|c|}{ After } & \multicolumn{1}{c|}{ iter. } \\
Region & Water Protein Adipose & Water Protein Adipose & Water Protein Adipose \\
\hline$R_{0}$ & 100 & 0 & 0 & -6.7 & 11.2 & 94.0 & 97.3 & 0.4 & 2.5 \\
$R_{1}$ & 0 & 100 & 0 & -58.8 & 104.1 & 56.3 & -1.4 & 100.0 & 1.5 \\
$R_{2}$ & 0 & 25 & 75 & -45.5 & 27.8 & 119.2 & 1.6 & 25.0 & 73.5 \\
$R_{3}$ & 30 & 40 & 30 & -64.9 & 46.4 & 118.0 & 31.9 & 39.9 & 28.2 \\
$R_{4}$ & 0 & 75 & 25 & -54.4 & 78.9 & 77.9 & 1.7 & 74.9 & 23.4 \\
$R_{5}$ & 0 & 0 & 100 & -41.3 & 2.9 & 139.1 & -2.4 & 0.5 & 101.7 \\
\hline
\end{tabular}

\subsection{Results}

The reconstruction results for the 0 th iteration (i.e. plain reconstruction without iterations) and the 7th iteration are given in Fig. 6. For comparison, the plain reconstructions from monoenergetic effective energies, $49,9 \mathrm{kV}$ and $88.5 \mathrm{kV}$, are also shown in the figure. Note that the results after 0 iterations are affected by beam hardening artifacts, whereas the results after 7 iterations are very similar to the monoenergetic reconstructions. The material decomposition results for the 0th iteration and the 7th iteration are given in Fig. 7 and Table 3 show mean values measured in a surrounding of each region. Note that the weight fractions are unaccepable after 0 iterations and sufficiently accurate after 7 iterations. 
80kV spectrum, 0 iter
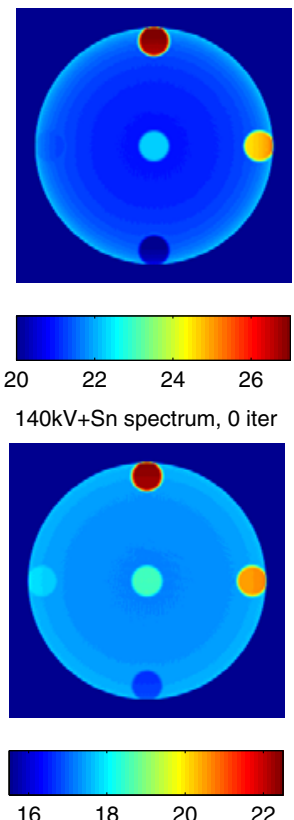

$80 \mathrm{kV}$ spectrum, 7 iter
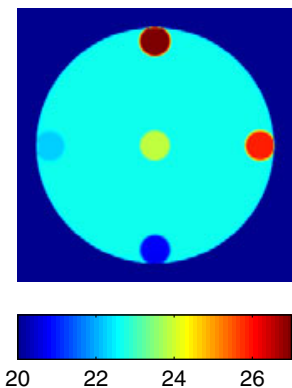

$140 k V+S n$ spectrum, 7 iter
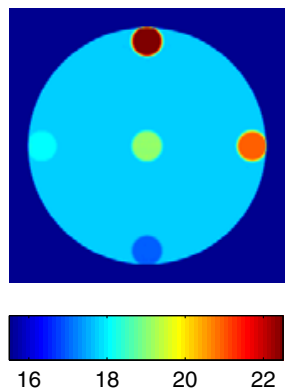

49.9keV mono
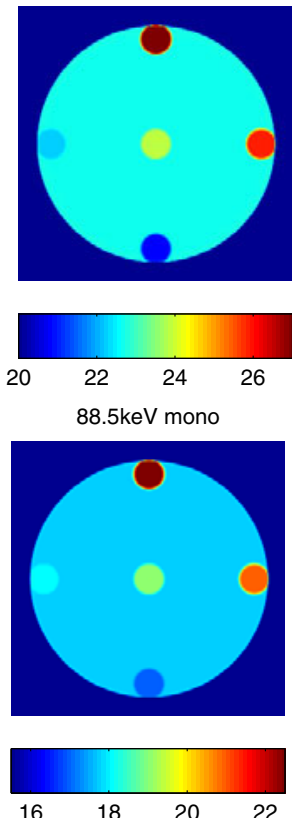

Fig. 6. Reconstruction results after 0 (left) and 7 (middle) iterations corresponding to $80 \mathrm{kV}$ (up) and $140 \mathrm{kV}+\mathrm{Sn}$ (down) spectrum. Right: Reconstruction results for monoenergetic effective energies, $49,9 \mathrm{kV}$ (up) and $88.5 \mathrm{kV}$ (down).
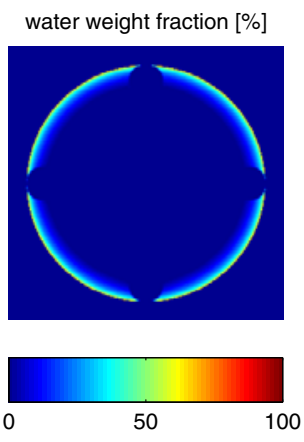

water weight fraction [\%]

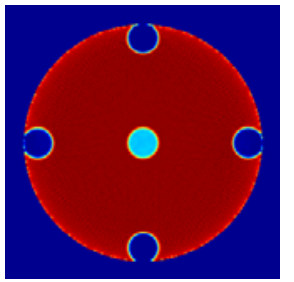

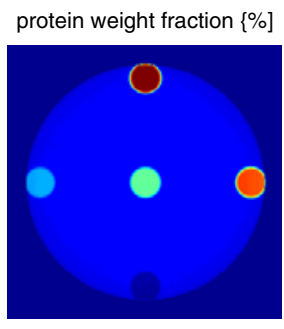
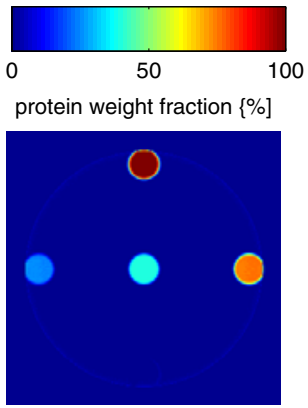

adipose weight fraction [\%]
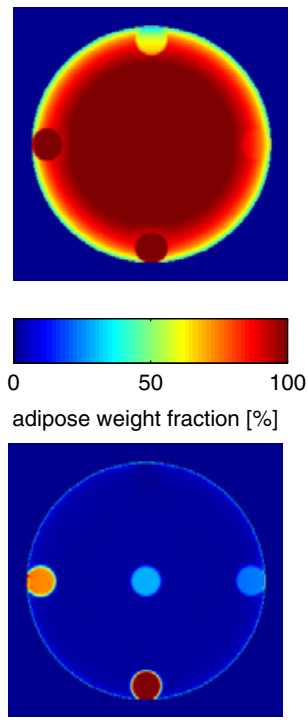

Fig. 7. Weight fractions for water, protein, adipose after 0 (up) and 7 (down) iterations 


\section{Conclusions and Future Work}

We have presented a novel iterative reconstruction algorithm for dual-energy $\mathrm{CT}$ that performs tissue decomposition and effectively removes beam hardening artifacts. The algorithm was evaluated using computer simulations with a cylindrical water phantom containing rod inserts consisting of mixtures of water, protein, and adipose tissue. The simulations demonstrated that the proposed iterative algorithm was able to accurately reconstruct mass fraction values of all base materials in the mixture.

In the near future we will improve our implementation with the aim to reduce the number of needed iterations ( 7 at the moment). Future plans also involves including other types of tissue, such as bone, applications on real measured CT data, and extension from 2-D images to 3-D data sets. Also, scatter and statistical noise will be considered and compensated for.

\section{References}

1. Kak, A.C., Slaney, M.: Principles of Computerized Tomographic Imaging. IEEE Press, Los Alamitos (1988)

2. Stierstorfer, K., Rauscher, A., Boese, J., Bruder, H., Schaller, S., Flohr, T.: Weighted FBP - a simple approximate 3D FBP algorithm for multislice spiral CT with good dose usage for arbitrary pitch. Phys. Med. Biol. 49, 2209-2218 (2004)

3. Persson, A., Jackowski, C., Engström, E., Zachrisson, H.: Advances of dual source, dual-energy imaging in postmortem CT. Eur. J. Radiol. 68, 446-455 (2008)

4. Schneider, W., Bortfeld, T., Schlegel, W.: Correlation between CT numbers and tissue parameters needed for Monte Carlo simulations of clinical dose distributions. Phys. Med. Biol. 45, 459-478 (2000)

5. Bazalova, M., Beaulieu, L., Palefsky, S., Verhaegen, F.: Tissue segmentation in Monte Carlo treatment planning: a simulation study using dual-energy CT images. Radiother Oncol. 86, 93-98 (2007)

6. Liu, X., Yu, L., Primak, A.N., McCollough, C.H.: Quantitative imaging of element composition and mass fraction using dual-energy CT: Three-material decomposition. Med. Phys. 36(5), 1602-1609 (2009)

7. Yu, L., Liu, X., McCollough, C.H.: Pre-reconstruction three-material decomposition in dual-energy CT. In: Proc. of SPIE, vol. 7258, pp. 72583V-1 (2009)

8. International Commission on Radiation Units and Measurements: Tissue Substitutes in Radiation Dosimetry and Measurement, ICRU Report No. 44 (1989)

9. De Man, B., Nuyts, J., Dupont, P., Marchal, G., Suetens, P.: An Iterative Maximum-Likelihood Polychromatic Algorithm for CT. IEEE Trans. on Med. Imaging 20(10) (2001)

10. Seger, O., Magnusson Seger, M.: The MATLAB/C program take - a program for simulation of X-ray projections from 3D volume data. Demonstration of beamhardening artifacts in subsequent CT reconstruction, Technical Report LiTH-ISYR-2682 (2005), http://liu.diva-portal.org

11. Joseph, P.: An Improved Algorithm for reprojectiong Rays Through Pixel Images. IEEE Trans. on Med. Imaging 1(3), 1992-1996 (1982)

12. National Institute of Standards and Technology, http://physics.nist.gov/PhysRefData/XrayMassCoef/cover.html 